OPEN ACCESS

Edited by:

Xiaotong Hou,

Capital Medical University, China

Reviewed by:

Pasquale Sansone

University of Campania Luigi

Vanvitelli, Italy

Yumei Li,

Jilin University, China

*Correspondence:

Chunxia Wang

karencx0465@163.com

Yucai Zhang

zyucai2018@163.com

${ }^{\dagger}$ These authors have contributed equally to this work

Specialty section This article was submitted to Intensive Care Medicine and

Anesthesiology

a section of the journal

Frontiers in Medicine

Received: 17 September 2021 Accepted: 31 December 2021 Published: 10 February 2022

Citation:

Wang C, Zheng J, Wang J, Zou L and Zhang Y (2022) Cox-LASSO Analysis for Hospital Mortality in Patients With Sepsis Received Continuous Renal Replacement Therapy: A MIMIC-III Database Study.

Front. Med. 8:778536. doi: 10.3389/fmed.2021.778536

\section{Cox-LASSO Analysis for Hospital Mortality in Patients With Sepsis Received Continuous Renal Replacement Therapy: A MIMIC-III Database Study}

\author{
Chunxia Wang ${ }^{1,2,3,4 *}$, Jianli Zheng ${ }^{5 t}$, Jinxia Wang ${ }^{3}$, Lin Zou ${ }^{3,4}$ and Yucai Zhang ${ }^{1,2,4 *}$
}

${ }^{1}$ Department of Critical Care Medicine, Shanghai Children's Hospital, Shanghai Jiao Tong University, Shanghai, China, ${ }^{2}$ Institute of Pediatric Critical Care, Shanghai Jiao Tong University, Shanghai, China, ${ }^{3}$ Clinical Research Unit, Shanghai Children's Hospital, Shanghai Jiao Tong University, Shanghai, China, ${ }^{4}$ Institute of Pediatric Infection, Immunity, and Critical Care Medicine, Shanghai Jiao Tong University School of Medicine, Shanghai, China, ${ }^{5}$ Institute of Medical Information Engineering, University of Shanghai for Science and Technology, Shanghai, China

Background: Sepsis remains the leading cause of mortality in-hospital in the intensive care unit (ICU). Continuous renal replacement therapy (CRRT) is recommended as an adjuvant therapy for hemodynamics management in patients with sepsis. The aim of this study was to develop an adaptive least absolute shrinkage and selection operator (LASSO) for the Cox regression model to predict the hospital mortality in patients with Sepsis-3.0 undergoing CRRT using Medical Information Martin Intensive Care (MIMIC)-III v1.4.

Methods: Patients who met the Sepsis-3.0 definition were identified using the MIMIC-III v1.4. Among them, patients who received CRRT during ICU hospitalization were included in this study. According to the survival status, patients were split into death or survival group. Adaptive LASSO for the Cox regression model was constructed by STATA software. At last, nomogram and Kaplan-Meier curves were drawn to validate the model.

Results: A total of 181 patients who met Sepsis 3.0 criteria received CRRT were included in the study, in which, there were 31 deaths and 150 survivals during hospitalization, respectively. The overall in-hospital mortality was $17.1 \%$. According to the results of multivariate Cox-LASSO regression analysis, use of vasopressor, international normalized ratio (INR) $\geq 1.5$, and quick sequential organ failure assessment (qSOFA) score were associated with hospital mortality in patients with sepsis who underwent CRRT, but lactate level, mechanical ventilation (MV) support, $\mathrm{PaO}_{2} / \mathrm{FiO}_{2}$, platelet count, and indicators of acute kidney injury (AKI), such as blood urea nitrogen (BUN) and creatinine, were not independently associated with hospital mortality after adjusted by qSOFA. The risk nomogram and Kaplan-Meier curves verified that the use of vasopressor and INR $\geq 1.5$ possess significant predictive value. 
Conclusions: Using the Cox-LASSO regression model, use of vasopressor, INR $\geq 1.5$, and QSOFA score are found to be associated with hospital mortality in patients with Sepsis-3.0 who received CRRT. This finding may assist clinicians in tailoring precise management and therapy for these patients who underwent CRRT.

Keywords: MIMIC-III, Sepsis-3.0, LASSO, Cox regression, mortality, CRRT

\section{INTRODUCTION}

Sepsis is a major condition with high morbidity and mortality in intensive care unit (ICU) patients (1). Severe sepsis and septic shock are characterized by vasoplegia and alterations of microcirculation, resulting in aggressively hemodynamic alterations that render the patient hypotensive or with organ dysfunction (2-5). During sepsis, fluid responsiveness or the use of vasopressors could guide fluid administration (6), but the response to therapy is highly variable $(7,8)$. Improvement of hemodynamic may not be related to the improvement of microcirculation $(4,9)$. Septic shock is defined as a microcirculation disease, and many trials showed that the severity of microvascular alterations is associated with outcomes in patients with septic shock (10-14). Evaluation of the response for hemodynamic management is critical for the prognosis of sepsis.

According to the 2020 Surviving Sepsis Campaign (SSC) guidelines, renal replacement therapy (RRT)/continuous RRT (CRRT) has emerged as the preferred modality for critically ill patients to treat acute kidney injury (AKI), fluid overload, particularly, those with hemodynamic instability who are unresponsive to fluid restriction and diuretic therapy (15). In adult septic patients who underwent RRT, microcirculation was improved despite no significant variation in macrohemodynamics (16). Sepsis-induced aggressively hemodynamic alterations are mainly caused by endothelial dysfunction resulting in the activation of inflammation and coagulation processes $(5,17)$. CRRT plays an important role in removing toxins and inflammatory factors, and higher TNF- $\alpha$ removal could be related to the lower mortality observed in patients with AKI (18). In addition, patients with sepsis suffer from a higher risk of bleeding and clotting. Anticoagulation is necessary for the effective delivery of CRRT, and anticoagulation for CRRT should be adapted to the patient's characteristics (19). Given the complex roles of CRRT in improving inflammatory response, fluid management, and anticoagulation involved in CRRT management, assessment of the prognosis in patients with sepsis who underwent CRRT could be especial. Until now,

\footnotetext{
Abbreviations: BP, blood pressure; BUN, blood urea nitrogen; $C I$, confidence intervals; CRRT, continuous renal replacement therapy; $H R$, hazard ratio; ICD, International Classification of Diseases; ICU, intensive care unit; INR, international normalized ratio; LODS, Logistic Organ Dysfunction System; MIMIC, Medical Information Mart for Intensive Care; $\mathrm{PaO}_{2} / \mathrm{FiO}_{2}$, the ratio of the partial pressure of oxygen in arterial blood $\left(\mathrm{PaO}_{2}\right)$ to the inspired oxygen fraction $\left(\mathrm{FiO}_{2}\right)$; qSOFA, quick sequential organ failure assessment; SIRS, systemic inflammatory response syndrome; SOFA, sequential organ failure assessment; $\mathrm{SpO}_{2}$, oxyhemoglobin saturation; SSC, surviving sepsis campaign; WBC, white blood cells.
}

the risk factors of worse prognosis in patients with sepsis who received CRRT are limited to be reported.

In the current study, we conducted a retrospective study based on Medical Information Martin Intensive Care (MIMIC) III v1.4 to develop a model based on the potential risk factors related to the outcome of patients with sepsis who need CRRT. The results could be helpful for clinicians to make precise management of these patients.

\section{METHODS}

\section{Database and Study Population}

Study data were acquired from the MIMIC-III database v1.4, which encompasses > 60,000 ICU admissions between 2001 and 2012 for $>46,000$ unique patients at Beth Israel Deaconess Medical Center (BIDMC) in Boston, Massachusetts between 2001 and 2012 (20). The information available in MIMIC-III includes dates of admission to the ICU and hospital, demographic, clinical features, laboratory and microbiology test results, fluid balance, critical illness scores, diagnosis codes, and hospital mortality. Use of the MIMIC-III database was approved by the Institutional Review Boards of BIDMC and the Massachusetts Institute of Technology.

Firstly, data extraction adhered to the original Sepsis-3.0 definition as closely as possible $(21,22)$. According to the report of Johnson (23), the patients who fulfilled the Sepsis-3.0 criteria were automatically extracted using pgAdmin PostgreSQL tools (version 1.22.1). Of these patients, patients who aged over 18year-old received CRRT during hospitalization were included. We excluded those with conditions who may be associated with hospital mortality, such as: (1) the length of ICU stay $<24 \mathrm{~h}$; (2) with chronic kidney disease (International Classification of Diseases [ICD]9-code: 5859); (3) metastatic cancer and solid tumor without metastasis (metastatic cancer: icd9_code: 1960-1991, 20970-20975, 20979, 78951; solid tumor without metastasis: icd9_code: 1400-1729, 1740-1759, 179-1958, 2090020924, 20925-2093, 20930-20936, 25801-25803); or (4) surgery plan. Patients were divided into two groups based on the record of the hospital expire flag (in-hospital death recorded in the hospital database). The detailed process of patients' selection and data extraction is shown in Figure 1.

\section{Outcomes}

The primary outcome was hospital mortality at the first ICU admission. The secondary outcomes were the length of ICU and hospital stay, use of vasopressor, and mechanical ventilation (MV) support. 


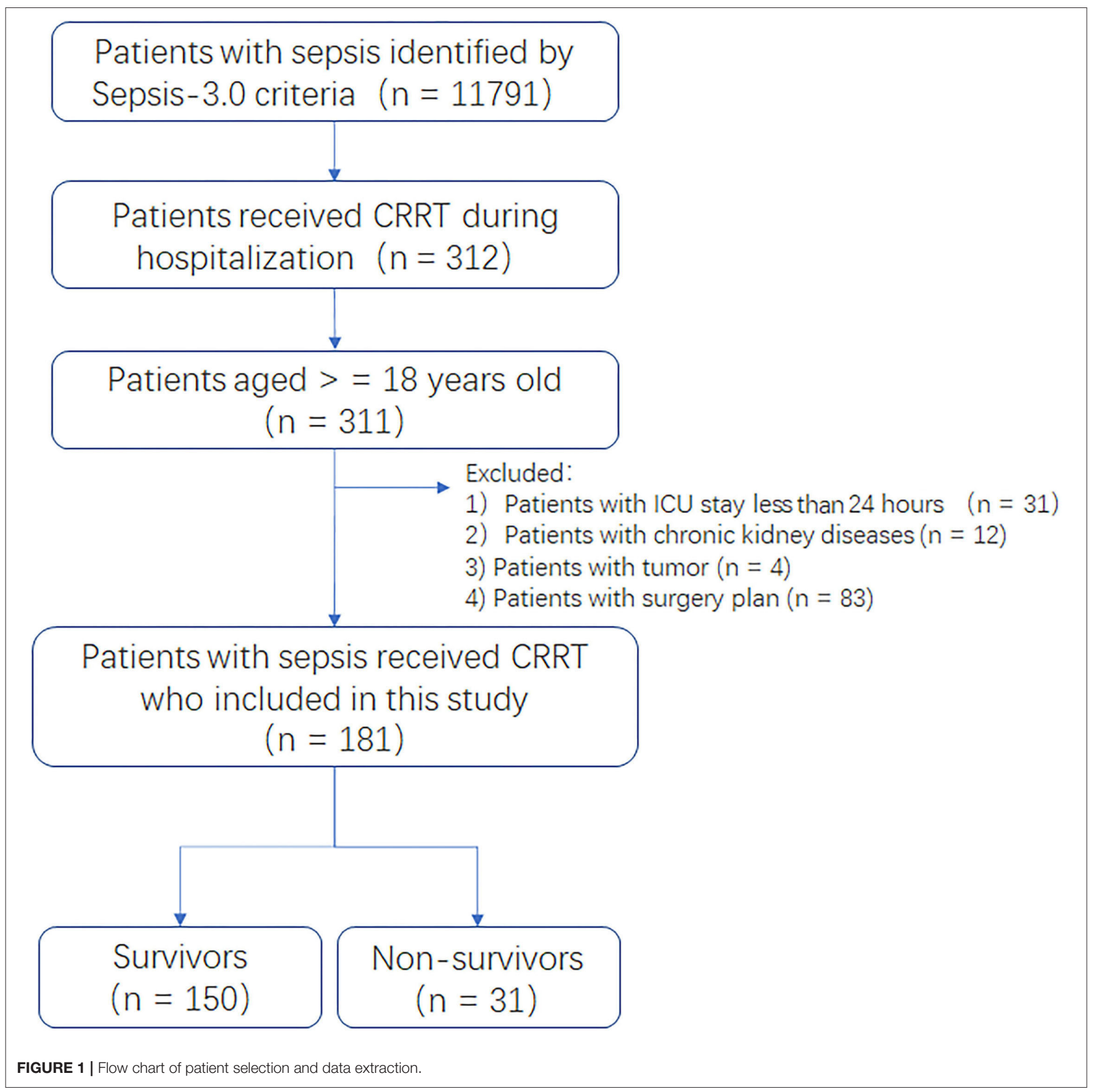

\section{Data Extraction and Variables Collection}

Variables extracted from MIMIC-III database v1.4 included demographics, severity accessed by SOFA, qSOFA, systemic inflammatory response syndrome (SIRS), and Logistic Organ Dysfunction System (LODS) scores, source of patients, vital signs, such as heart rate (HR), systolic blood pressure (BP), diastolic $\mathrm{BP}$, mean arterial pressure (MAP), temperature, respiratory rate (RR), arterial blood gas, such as oxyhemoglobin saturation $\left(\mathrm{SpO}_{2}\right)$ and $\mathrm{PaO}_{2} / \mathrm{FiO}_{2}$, serum laboratory variables that include the minimum of albumin, platelet, the maximum of bilirubin, creatinine, lactate, international normalized ratio (INR), blood urea nitrogen (BUN), and white blood cells (WBC), and the test results of blood infection. Furthermore, oxygen therapy support mode, duration of ventilation, use of vasopressor, and vasopressor duration were accessed. Patient demographics and all necessary variables were calculated using data from the first $24 \mathrm{~h}$ of the ICU stay. Furthermore, we set categorical variables based on the values of laboratory indexes within $24 \mathrm{~h}$ after ICU admission as below: (1) systolic $\mathrm{BP}<100 \mathrm{mmHg}$, (2) whether or not need vasopressor, (3) INR $\geq 1.5$, (4) platelet $<100 \times 10^{9} / \mathrm{L}$, (5) 
TABLE 1 | Baseline characteristics in patients with sepsis who received CRRT.

\begin{tabular}{|c|c|c|c|c|}
\hline Parameters & Total $(n=181)$ & Survivors $(n=150)$ & Non-survivors $(n=31)$ & $P$ \\
\hline \multicolumn{5}{|l|}{ Demographic variables } \\
\hline Gender male, $n(\%)$ & 109 (60.2) & 89 (59.3) & $20(64.5)$ & 0.591 \\
\hline Age, year, mean (SD) & $61.9(14.82)$ & $61.2(15.11)$ & $65.0(13.12)$ & 0.196 \\
\hline \multicolumn{5}{|l|}{ Ethnicity, n 0.530} \\
\hline White & 101 & 81 & 20 & 0.283 \\
\hline Black & 34 & 32 & 2 & 0.053 \\
\hline Hispanic & 12 & 11 & 1 & 0.403 \\
\hline Others & 34 & 26 & 8 & 0.272 \\
\hline \multicolumn{5}{|l|}{ Severity, median (IQR) } \\
\hline SOFA & $7(5-10)$ & $6(5-9)$ & $11(8-16)$ & $<0.001$ \\
\hline qSOFA & $2(1-2)$ & $2(1-2)$ & $2(2-3)$ & $<0.001$ \\
\hline SIRS & $3(2-4)$ & $3(2-3)$ & $3(2-4)$ & 0.033 \\
\hline LODS & $6(5-8)$ & $6(4-7)$ & $9(7-13)$ & $<0.001$ \\
\hline First service, $n$ & & & & 0.546 \\
\hline CMED & 24 & 18 & 6 & \\
\hline MED & 152 & 127 & 25 & \\
\hline NMED & 2 & 2 & 0 & \\
\hline OMED & 3 & 3 & 0 & \\
\hline Blood infection, $n$ & 68 & 54 & 14 & 0.338 \\
\hline Mechanical ventilation, $n(\%)$ & $76(42)$ & $53(35.3)$ & $23(74.2)$ & $<0.001$ \\
\hline Length of ICU stay, days, median (IQR) & $2.9(1.8-5.7)$ & $2.8(1.8-5.7)$ & $3.3(1.7-7.2)$ & 0.778 \\
\hline Length of hospital stay, days, median (IQR) & $7.7(4.1-4.0)$ & $9.8(5.1-15.6)$ & $3.4(1.6-9.8)$ & $<0.001$ \\
\hline
\end{tabular}

SOFA, sequential organ failure assessment; qSOFA, Quick SOFA; SIRS, systemic inflammatory response syndrome; LODS, Logistic Organ Dysfunction System.

lactate $\geq 4 \mu \mathrm{mol} / \mathrm{L}$, (6) impaired pulmonary function was defined as $\mathrm{PaO}_{2} / \mathrm{FiO}_{2}>300 \mathrm{mmHg}, 200 \mathrm{mmHg}<\mathrm{PaO}_{2} / \mathrm{FiO}_{2} \leq 300$ $\mathrm{mmHg}, 100 \mathrm{mmHg}<\mathrm{PaO}_{2} / \mathrm{FiO}_{2} \leq 200 \mathrm{mmHg}$, and $\mathrm{PaO}_{2} / \mathrm{FiO}_{2}$ $\leq 100 \mathrm{mmHg}$. Ultimately, we obtained the list of data based on anonymized patients with Sepsis-3.0 who received CRRT.

\section{Statistical Analysis}

All the data analyses were conducted using STATA 15.0 MP (College Station, TX, USA). Variables were displayed and compared between survivors and non-survivors. Normally and non-normally distributed continuous variables were expressed as the mean $\pm \mathrm{SD}$ and the median (interquartile range, IQR), respectively. Continuous variables of normal distribution were tested by Student's $t$-test. Mann-Whitney $U$-test was used to compare continuous data of non-normally distribution. Categorical variables were summarized as numbers or percentage and assessed using the Chi-square test. A $p<0.05$ was defined as statistically significant.

Cox survival analysis and least absolute shrinkage and selection operator (LASSO) regression univariable regression analyses were performed to assess the association of each variable with hospital mortality, and values of $p<0.05$ were selected as a candidate variable. The method of LASSO was used to select predictors. Multivariate Cox regression was further performed to assess the prognostic value of selected variables, with qSOFA as an adjustment factor. The hazard ratio (HR) and 95\% CI were estimated by Cox proportional hazards regression model.

\section{Construction and Validation of a Prognostic Nomogram for Hospital Mortality}

Nomogram were constructed to calculate an individual's probability of hospital mortality by using STATA software. In the nomogram, the patient was scored according to the variables entered multivariate Cox proportional hazards regression model. The final sum of the scores was expected to be the corresponding hospital mortality probability. Kaplan-Meier curves were drawn and compared the differences in hospital mortality between groups divided by the variables of the nomogram.

\section{RESULTS}

\section{Baseline Characteristics}

There were 11,791 patients with Sepsis-3.0 between 2008 and 2012. In this cohort, 312 patients received CRRT during hospitalization. One patient aged less 18-year-old, 31 cases with the length of ICU stay $<24 \mathrm{~h}, 12$ patients with chronic kidney disease, 4 patients with tumor, and 83 patients with surgery plan were excluded. Finally, there were 181 patients with Sepsis-3.0 who underwent CRRT during hospitalization, in which, there were 31 deaths and 150 survivals during hospitalization, respectively.

In these patients, the age, gender, ethnicity, first service type, blood infection, and the length of ICU stay showed no significant difference between survival and non-survival groups. The ratio of 
TABLE 2 | Laboratory indexes within $24 \mathrm{~h}$ after ICU admission in patients with sepsis who received CRRT.

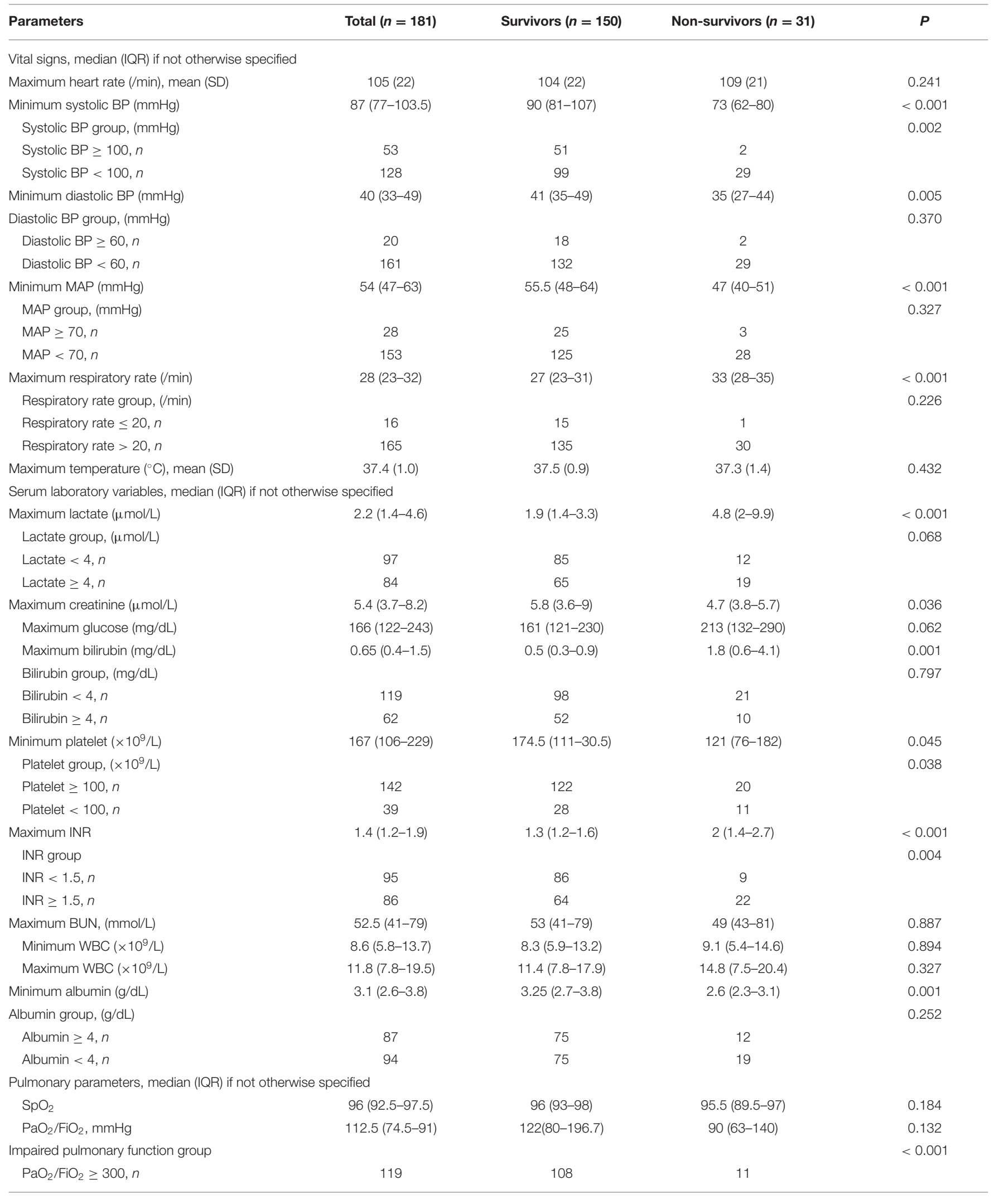


TABLE 2 | Continued

\begin{tabular}{|c|c|c|c|c|}
\hline Parameters & Total $(n=181)$ & Survivors $(n=150)$ & Non-survivors $(n=31)$ & $P$ \\
\hline $300<\mathrm{PaO}_{2} / \mathrm{FiO} 2 \leq 200, n$ & 8 & 5 & 5 & \\
\hline $200<\mathrm{PaO}_{2} / \mathrm{FiO}_{2} \leq 100, n$ & 25 & 20 & 5 & \\
\hline Vasopressor & & & & $<0.001$ \\
\hline No, $n(\%)$ & $109(60.2)$ & $104(69.3)$ & $5(16.1)$ & \\
\hline Respiratory support model, $n$ (\%) & & & & $<0.001$ \\
\hline None, $n(\%)$ & 19 (10.5) & $19(12.7)$ & $0(0)$ & \\
\hline Oxygen therapy, $n$ (\%) & $82(45.3)$ & $76(50.7)$ & $6(19.4)$ & \\
\hline Mechanical ventilation, $n(\%)$ & $80(44.2)$ & $55(36.7)$ & $25(80.6)$ & \\
\hline Ventilation durations, hours & $65.6(27.9-67.5)$ & $82(30.8-89.8)$ & $60(27.4-98.2)$ & 0.338 \\
\hline
\end{tabular}

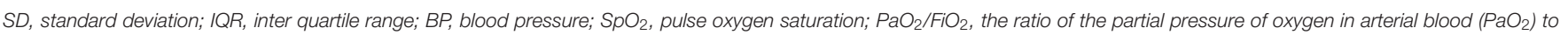
the inspired oxygen fraction $\left(\mathrm{FiO}_{2}\right)$; INR, international normalized ratio.

MV was higher in non-survivors than survivors, and the length of hospital stay was shorter in non-survivors than survivors (Table 1).

\section{Laboratory Indexes Within the First $24 \mathrm{~h}$ After ICU Admission}

Minimum systolic BP, minimum diastolic BP, minimum MAP, maximum RR, maximum lactate, maximum creatinine, maximum bilirubin, minimum platelet, maximum INR, minimum albumin, ratio of vasopressor needed, and respiratory support were significantly different between survivors and non-survivors. However, maximum $\mathrm{HR}$, maximum temperature, maximum glucose, maximum BUN, minimum WBC, maximum WBC, $\mathrm{SpO}_{2}, \mathrm{PaO}_{2} / \mathrm{FiO}_{2}$, vasopressor duration, and ventilation durations showed no significant difference between the two groups (Table 2 ).

\section{Relationship Between Clinical and Laboratory Indexes and Hospital Mortality}

Overall hospital mortality was $17.1 \%$ (31/181). The ratio of MV needed was $44.2 \%(80 / 181)$, and the ratio of use of vasopressor was $39.8 \%(72 / 181)$. The hospital mortality was $36.1 \%(26 / 72)$ in patients who received vasopressor and $4.6 \%(5 / 109)$ in patients without vasopressor support $(p<0.001)$. In a subgroup of patients who received MV support, the hospital mortality was $31.3(25 / 80)$, which was significantly higher than that $7.3 \%(6 / 82)$ in patients with oxygen therapy, and all 19 patients without any oxygen therapy were survival.

\section{Identification of Risk Factors of Hospital Mortality by Cox-LASSO Analysis}

According to the results of Table 2, laboratory variables and categorical variables with statistically significant differences between survivors and non-survivors were entered in the Univariate Cox analysis. The results showed that systolic BP $<$ $100 \mathrm{mmHg}$, the use of vasopressor, INR $\geq 1.5$, maximum lactate, maximum creatinine, and impaired severity of pulmonary
TABLE 3 | Univariate Cox analysis of factor related to hospital mortality in patients with sepsis received CRRT.

\begin{tabular}{|c|c|c|}
\hline Parameters & $H R(95 \%$ Cl) & $P$ \\
\hline Minimum systolic BP & $0.956(0.938-0.976)$ & $<0.001$ \\
\hline Systolic BP $<100$ mmHg & $5.327(1.268-22.388)$ & 0.022 \\
\hline Use of vasopressor & $6,860(2.622-17.947)$ & $<0.001$ \\
\hline Maximum INR & $1.231(1.127-1.346)$ & $<0.001$ \\
\hline$I N R \geq 1.5$ & $2.639(1.208-5.767)$ & 0.015 \\
\hline Minimum platelet & $0.998(0.995-1.001)$ & 0.270 \\
\hline Platelet $<100 * 10^{9} / \mathrm{L}$ & $1.890(0.901-3.967)$ & 0.092 \\
\hline Maximum lactate & $1.140(1.071-1.213)$ & $<0.001$ \\
\hline Lactate $\geq 4 \mu \mathrm{mol} / \mathrm{L}$ & $2.017(0.978-4.158)$ & 0.057 \\
\hline Maximum creatinine & $0.873(0.772-0.987)$ & 0.031 \\
\hline $\mathrm{PaO}_{2} / \mathrm{FiO}_{2}$ & $0.997(0.991-1.002)$ & 0.244 \\
\hline Severity of impaired pulmonary function & $1.580(1.203-2.076)$ & 0.001 \\
\hline qSOFA & $2.523(1.455-4.374)$ & 0.001 \\
\hline
\end{tabular}

$\mathrm{HR}$, hazard ratio; $\mathrm{Cl}$, confidence interval; $\mathrm{BP}$, blood pressure; $\mathrm{PaO}_{2} / \mathrm{FiO}_{2}$, the ratio of the partial pressure of oxygen in arterial blood $\left(\mathrm{PaO}_{2}\right)$ to the inspired oxygen fraction $\left(\mathrm{FiO}_{2}\right)$; INR, International normalized ratio; qSOFA, quick sequential organ failure assessment.

function were associated with hospital mortality in patients with sepsis undergoing CRRT (all $p<0.05$; Table 3). Furthermore, $L A S S O$ regression analysis was used to screen these variables. Adaptive LASSO regression analysis indicated that the categorical variables, such as the use of vasopressor, INR $\geq 1.5$, impaired severity of pulmonary function, but not the absolute values of laboratory indexes, were entered multivariate Cox regression model (Figure 2). Finally, multivariate Cox regression model based on the adaptive LASSO displayed that the use of vasopressor and INR $\geq 1.5$ were risk factors of hospital mortality in patients with Sepsis-3.0 who received CRRT adjusted by qSOFA (Table 4). 


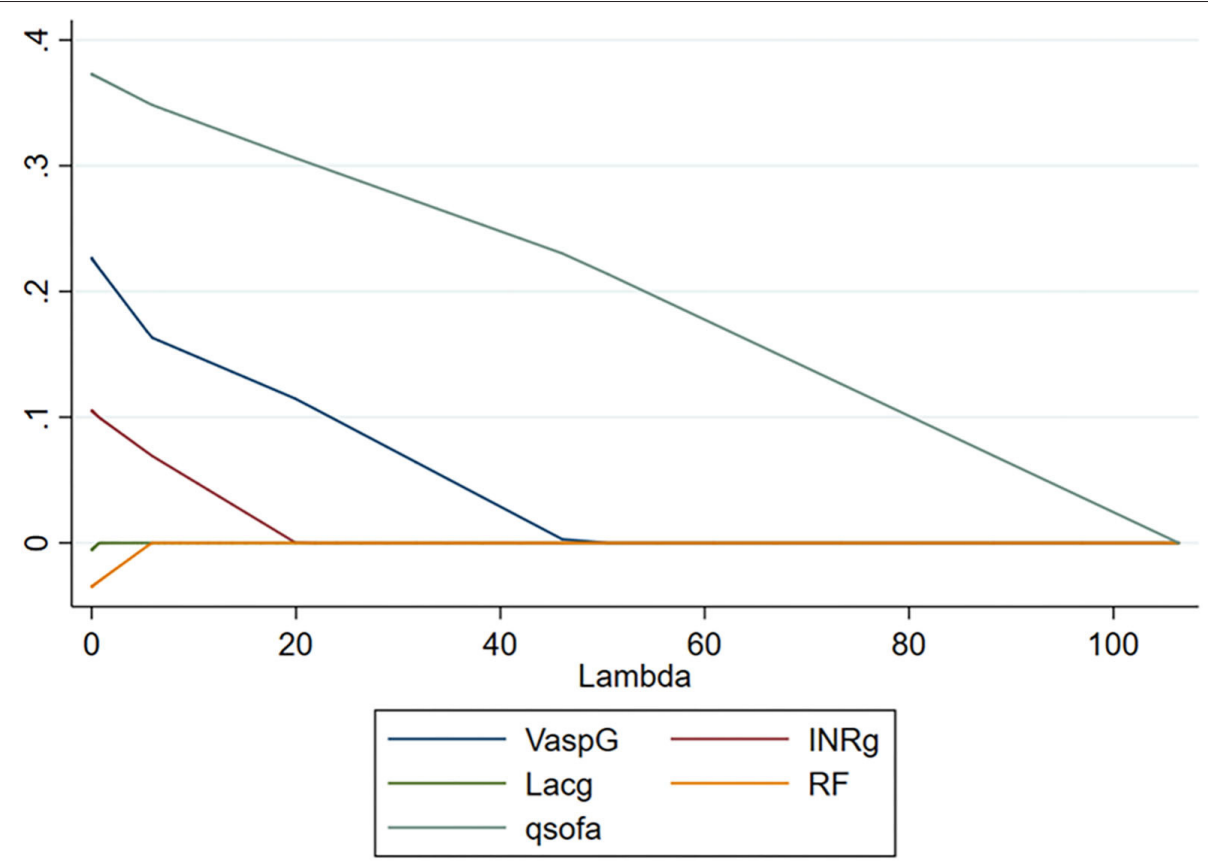

FIGURE 2 | LASSO regression analysis for hospital mortality in patients with Sepsis-3.0 who received CRRT. VaspG (blue line): use of vasopressor; INRg (purple line): INR > 1.5; Lacg (green line): lactate $\geq 4 \mu \mathrm{mol} / \mathrm{L} ; \mathrm{RF}$ (orange line): severity of impaired pulmonary function $\left(\right.$ defined as $\mathrm{PaO}_{2} / \mathrm{FiO}{ }_{2}(\mathrm{PF})>300 \mathrm{mmHg}, 200 \mathrm{mmHg}<\mathrm{PF} \leq$ $300 \mathrm{mmHg}, 100 \mathrm{mmHg}<\mathrm{PF} \leq 200 \mathrm{mmHg}$, PF $\leq 100 \mathrm{mmHg}$ ); qSOFA (dark green line): qSOFA score. LASSO, least absolute shrinkage and selection operator; CRRT, continuous renal replacement therapy; qSOFA, quick sequential organ failure assessment; INR, international normalized ratio.

\section{A New Prognostic Nomogram for Patients With Sepsis-3.0 Who Underwent CRRT}

To provide a quantitative method for clinical outcome prediction, we constructed a prognostic nomogram, such as the use of vasopressor, INR $\geq 1.5$, the severity of impaired pulmonary function, and qSOFA, to predict the hospital mortality of patients with Sepsis-3.0. As shown in Figure 3, total scores were derived from the sum of the individual scores of various risk factors. In this nomogram, a higher total number of points indicated worse hospital mortality.

\section{Stratified Analysis of Prognostic Factors Using Kaplan-Meier Curves}

Further, we evaluated the prognostic value of the use of vasopressor, INR $>1.5$, the severity of impaired pulmonary function, and qSOFA score for the patients with Sepsis-3.0 who received CRRT. A significant difference in clinical outcomes was observed between with and without vasopressor support (Figure 4A, $p<0.001$ ), INR $>1.5$ compared with INR $\leq 1.5$ (Figure 4B, $p=0.012$ ), among different severity of impaired pulmonary function indicated with the value of $\mathrm{PaO}_{2} / \mathrm{FiO}_{2}$ (Figure 4C, $p<0.001$ ), and with or without MV support (Figure $4 \mathrm{D}, p<0.001$ ).

\section{DISCUSSION}

Sepsis-induced aggressive hemodynamic alterations are one of the main causes for high mortality in patients with sepsis. CRRT,
TABLE 4 | Multivariate Cox analysis of factor related to hospital mortality based on LASSO regression in patients with sepsis received CRRT.

\begin{tabular}{lcc}
\hline Parameters & HR (95\% $\mathbf{C l})$ & $\boldsymbol{P}$ \\
\hline Use of vasopressor & $4.564(1.575-13.223)$ & 0.005 \\
INR $\geq 1.5$ & $2.475(1.114-5.497)$ & 0.026 \\
Severity of impaired pulmonary function & $1.066(0.782-1.454)$ & 0.685 \\
qSOFA & $2.514(1.322-4.780)$ & 0.005
\end{tabular}

HR, hazard ratio; Cl, confidence interval; INR, International normalized ratio; qSOFA, quick sequential organ failure assessment; Continuous renal replacement therapy.

as a recommended management for hemodynamic stable, is paid more attention in recent years. In the present study, the retrospective study based on MIMIC-III v1.4 developed a CoxLASSO model to show that use of vasopressor and INR $\geq 1.5$ are found to be risk factors of hospital mortality in patients with sepsis who received CRRT. These findings may assist clinicians in tailoring precise management and therapy for these patients who underwent CRRT.

According to the international guideline for the management of sepsis in 2016 (24), CRRT is suggested to be used to facilitate the management of fluid balance in hemodynamically unstable septic patients. In the present study, the ratio of CRRT support in patients who met the criteria of Sepsis-3.0 was 2.6\% (312/11791). There were about $5-6 \%$ of ICU patients with AKI who will receive RRT (25). This result is much lower than the ratio of CRRT application in patients with sepsis in adult ICU in China (16.3\%) (26) and in pediatric ICU according to our previous 


\section{Nomogram}

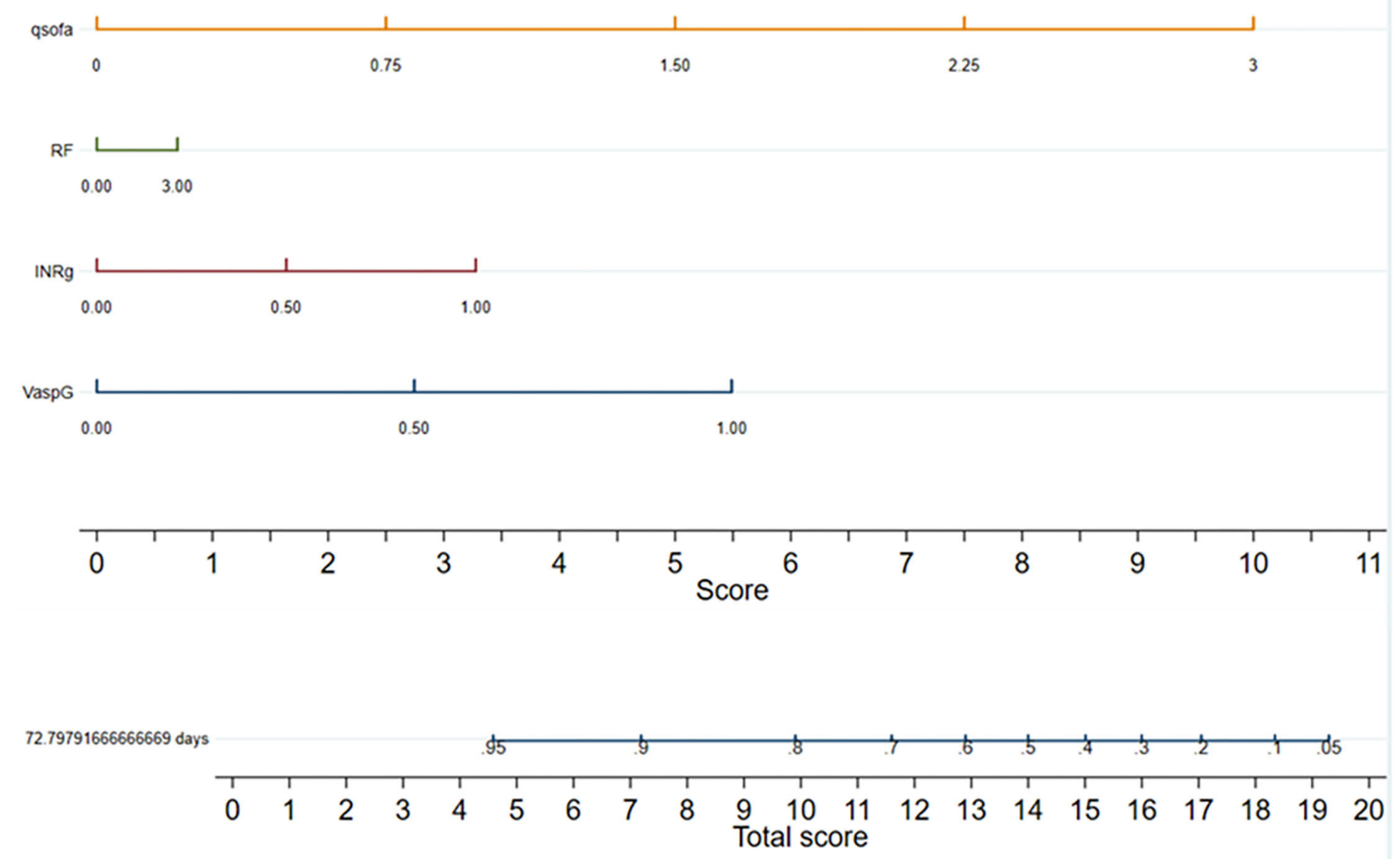

FIGURE 3 | Top features were selected using multivariate Cox-LASSO regression analysis and the corresponding variable importance score. The X-axis indicates the importance score, which is the relative number of a variable that is used to distribute the data, the $Y$-axis indicates the top-weighted variables. qSOFA (orange line): quick sequential organ failure assessment; $\mathrm{RF}$ (green line): impaired severity of pulmonary function (defined as $\left.\mathrm{PaO}_{2} / \mathrm{FiO} 2[\mathrm{PF}]\right)>300 \mathrm{mmHg}, 200 \mathrm{mmHg}<\mathrm{PF} \leq 300$ $\mathrm{mmHg}, 100 \mathrm{mmHg}<\mathrm{PF} \leq 200 \mathrm{mmHg}$, PF $\leq 100 \mathrm{mmHg}$ ). INRg (purple line): INR > 1.5; VaspG (blue line): use of vasopressor. LASSO, least absolute shrinkage and selection operator; CRRT, continuous renal replacement therapy; qSOFA, quick sequential organ failure assessment; INR, international normalized ratio.

study (10.7\%) (27). As reported, the ratio of CRRT used in a patient with COVID-19-induced that AKI was from 3.9\% $(225 / 5700)$ to $8.7 \%(280 / 3235)$ in the USA (28). MIMIC-III v1.4 did not include detailed information about the indications for CRRT in each patient. According to the baseline characteristics of patients with sepsis who received CRRT, there were high ratio $(70.7 \%, 128 / 181)$ of systolic $\mathrm{BP}<100 \mathrm{mmHg}$, high lactate levels $(\geq 4 \mu \mathrm{mol} / \mathrm{L})(46.4 \%, 84 / 181)$, high ratio of vasopressor support $(39.8 \%, 72 / 181)$, and a high ratio of MV support (44.2\%, 80/181). Miao et al. (29) reported the indications of CRRT in pediatric severe sepsis (http://links.lww.com/CCM/ E733). Whether there are more detailed differences in CRRT application between pediatric and adult patients with sepsis who need further investigation in a well-designed study.

In adult ICU, hospital mortality and 28-day mortality ranged from 50.4 to $64.5 \%$ in patients with septic AKI under CRRT $(30,31)$. The recent insights and results from large randomized and non-randomized trials in the area of RRT applied for sepsis-induced AKI do not seem to improve survival or renal recovery (32). Our previous study indicated that CRRT decreases hospital mortality rate from $32.4 \%(44 / 136)$ to $21.3 \%(29 / 136)$ in pediatric patients with severe sepsis (29). Moreover, there is no evidence to indicate that high-volume hemofiltration (HVHF) is superior to standard-volume continuous venovenous hemofiltration $(\mathrm{CVVH})$ in the aspect of reducing 28-day mortality in pediatric patients with severe sepsis ( 24.7 vs. $33.8 \%$, $p=0.216$ ) (33). In our present study, the total hospital mortality was $17.1 \%(31 / 181)$. In the subgroup, the hospital mortality was $22.6 \%(19 / 84)$ in patients with lactate $\geq 4 \mu \mathrm{mol} / \mathrm{L}, 22.6 \%$ (29/128) with systolic BP $<100 \mathrm{mmHg}, 31.3 \%$ with MV support, and $36.1 \%(26 / 72)$ with vasopressor support. These findings give an overview of the clinical benefits of CRRT in adult sepsis.

Hypotension or the need for vasoactive drugs was associated with mortality (34). In our present study, the need for vasoactive drugs, but not hypotension (defined as systolic $\mathrm{BP}<100 \mathrm{mmHg}$ ), on ICU admission was entered the LASSO model for mortality in a patient with sepsis under CRRT. In addition, thrombocytopenia prior to the initiation of CRRT and severe thrombocytopenia 
A

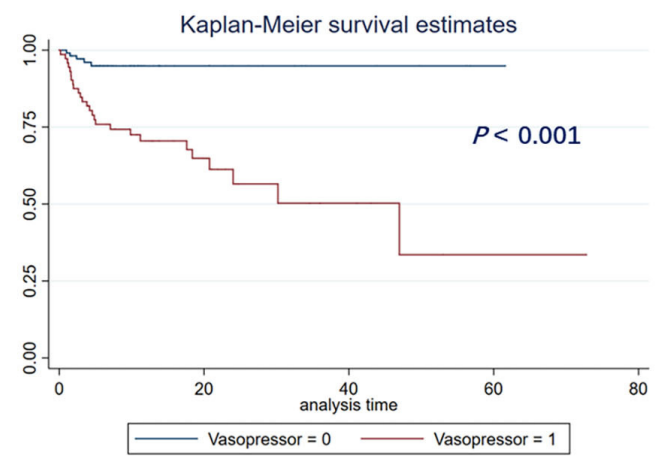

C

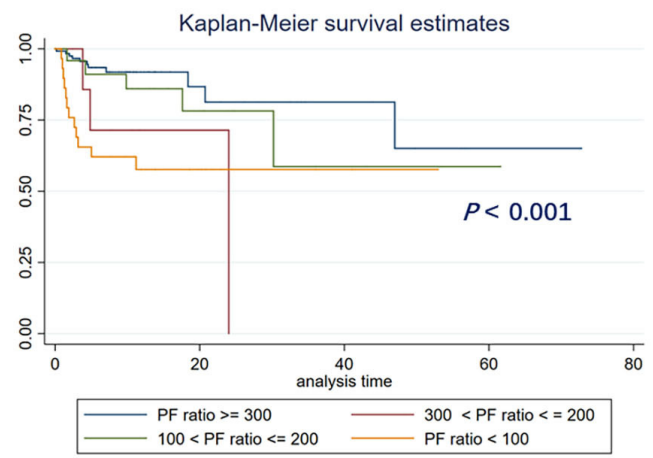

B

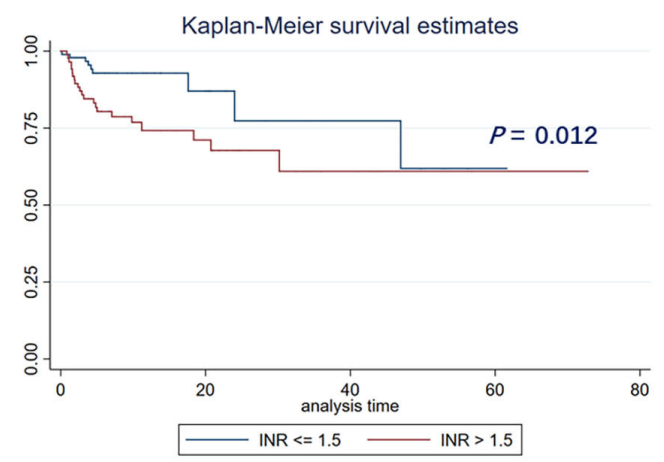

D

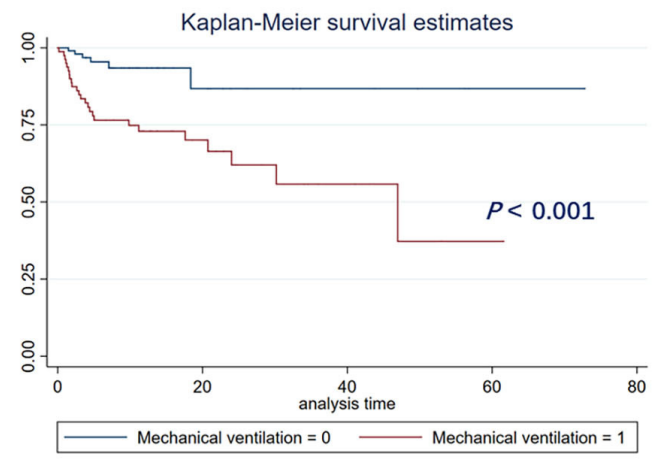

FIGURE 4 | Kaplan-Meier survival curve analysis of hospital mortality in patients stratified by prognostic factors based on Cox-LASSO analysis. (A) With vs. without vasopressor; (B) INF $\leq 1.5$ vs. INR $>1.5$; (C) $\mathrm{PaO}_{2} / \mathrm{FiO}_{2}$ (PF) $>300 \mathrm{mmHg}$ vs. $200 \mathrm{mmHg}<\mathrm{PF} \leq 300 \mathrm{mmHg}$ vs. $100 \mathrm{mmHg}<\mathrm{PF} \leq 200 \mathrm{mmHg}$ vs. PF $\leq 100$ $\mathrm{mmHg}$ ); (D) with or without mechanical ventilation support. LASSO, least absolute shrinkage and selection operator; INR, international normalized ratio.

prior to and following the initiation of CRRT were associated with increased ICU mortality (35). In the present study, though the ratio of patients with platelets $<100 \times 10^{9} / \mathrm{L}$ was higher in non-survivors than survivors, the ratio of patients with platelets $<100 \times 10^{9} / \mathrm{L}$ on admission was not an independent factor for mortality in patients under CRRT support. Moreover, AKI is a main indication for CRRT initiation, but the levels of serum creatinine were relatively lower in non-survivors than survivors, and there were no differences in the levels of BUN between the two groups. Consistently, there was a report that the severity of the AKI at the time of CRRT start did not have a significant relationship with the burned patient outcome with CRRT (36). Otherwise, in sepsis patients with AKI treated with CRRT, age, Acute Physiology and Chronic Health Evaluation (APACHE) II, SOAF, and grade IV of cardiac function were independent risk factors for death (37). In this study, qSOFA score was associated with mortality in patients treated with CRRT.

There are several limitations in this study. Firstly, we could not collect the detailed information about fluid overload in patients with sepsis. Secondly, the indications for CRRT were lacking in this study. Thirdly, as a database study, the interval time between sepsis occurrence and CRRT initiation was lacking. All these limitations could lead to bias for the present conclusions of this study, which needs further confirmation in a well-designed prospective study.

\section{CONCLUSIONS}

In summary, we found that the use of vasopressor, INR $\geq 1.5$, and qSOFA score are outcome of patients with sepsis who received CRRT based on MIMIC-III v1.4. After adjusted by qSOFA score, either lactate level or MV support is independently associated with the hospital mortality. These findings may assist clinicians in tailoring precise management of hemodynamics and coagulation disorders for these patients who underwent CRRT.

\section{DATA AVAILABILITY STATEMENT}

The datasets presented in this study can be found in online repositories. The names of the repository/repositories and accession number(s) can be found in the article/Supplementary Material.

\section{ETHICS STATEMENT}

Ethical review and approval was not required for the study on human participants in accordance with the local legislation and institutional requirements. Written informed consent for participation was not required for this study in accordance with the national legislation and the institutional requirements. 


\section{AUTHOR CONTRIBUTIONS}

CW and YZ conceptualized the research aims. CW planned the analyses, guided the literature review, and drafted the manuscript. JZ extracted the data from the MIMIC-III database. $\mathrm{CW}, \mathrm{JZ}$, and JW participated in processing the data and doing the statistical analysis. LZ and YZ provided comments and approved the final manuscript. All authors read and approved the final manuscript.

\section{FUNDING}

This work was supported by the National Natural Science Foundation of China (82171729), the Natural

\section{REFERENCES}

1. Rudd KE, Johnson SC, Agesa KM, Shackelford KA, Tsoi D, Kievlan DR, et al. Global, regional, and national sepsis incidence and mortality, 1990-2017: analysis for the Global Burden of Disease Study. Lancet. (2020) 395:20011. doi: 10.1016/S0140-6736(19)32989-7

2. Pan $\mathrm{P}, \mathrm{Su}$ L, Liu D, Wang $X$. Microcirculation-guided protection strategy in hemodynamic therapy. Clin Hemorheol Microcirc. (2020) 75:24353. doi: $10.3233 / \mathrm{CH}-190784$

3. Puntillo F, Giglio M, Pasqualucci A, Brienza N, Paladini A, Varrassi G. Vasopressor-sparing action of methylene blue in severe sepsis and shock: a narrative review. Adv Ther. (2020) 37:3692-706. doi: 10.1007/s12325-020-01422-x

4. Scheuzger J, Zehnder A, Meier V, Yeginsoy D, Flukiger J, Siegemund M. Sublingual microcirculation does not reflect red blood cell transfusion thresholds in the intensive care unit-a prospective observational study in the intensive care unit. Crit Care. (2020) 24:18. doi: 10.1186/s13054-020-2728-7

5. De Backer D, Ricottilli F, Ospina-Tascon GA. Septic shock: a microcirculation disease. Curr Opin Anaesthesiol. (2021) 34:85-91. doi: 10.1097/ACO.0000000000000957

6. Douglas IS, Alapat PM, Corl KA, Exline MC, Forni LG, Holder AL, et al. Fluid response evaluation in sepsis hypotension and shock: a randomized clinical trial. Chest. (2020) 158:1431-45. doi: 10.1016/j.chest.2020.04.025

7. Potter EK, Hodgson L, Creagh-Brown B, Forni LG. Manipulating the microcirculation in sepsis - the impact of vasoactive medications on microcirculatory blood flow: a systematic review. Shock. (2019) 52:512. doi: 10.1097/SHK.0000000000001239

8. van Loon LM, van der Hoeven JG, Lemson J. Hemodynamic response to betablockers in severe sepsis and septic shock: a review of current literature. J Crit Care. (2019) 50:138-43. doi: 10.1016/j.jcrc.2018.12.003

9. Collet M, Huot B, Barthelemy R, Damoisel C, Payen D, Mebazaa A, et al. Influence of systemic hemodynamics on microcirculation during sepsis. J Crit Care. (2019) 52:213-8. doi: 10.1016/j.jcrc.2019.05.002

10. Pranskunas A, Koopmans M, Koetsier PM, Pilvinis V, Boerma EC. Microcirculatory blood flow as a tool to select ICU patients eligible for fluid therapy. Intensive Care Med. (2013) 39:612-9. doi: 10.1007/s00134-012-2793-8

11. Massey MJ, Hou PC, Filbin M, Wang H, Ngo L, Huang DT, et al. Microcirculatory perfusion disturbances in septic shock: results from the ProCESS trial. Crit Care. (2018) 22:308. doi: 10.1186/s13054-0182240-5

12. Scorcella C, Damiani E, Domizi R, Pierantozzi S, Tondi S, Carsetti A, et al. MicroDAIMON study: Microcirculatory DAIly MONitoring in critically ill patients: a prospective observational study. Ann Intensive Care. (2018) 8:64. doi: 10.1186/s13613-018-0411-9

13. Kazune S, Grabovskis A, Cescon C, Strike E, Vanags I. Association between increased arterial stiffness and clinical outcomes in patients with early sepsis:
Science Foundation of Shanghai (19ZR1442500), and National Key R\&D Program of China (2020YFC2005802 and 2020YFC2005800). CW was funded by the Shanghai Municipal Education Commission-Gaofeng Clinical Medicine Grant (20171928) and the talent program from Shanghai Jiao Tong University School of Medicine (17XJ11018).

\section{SUPPLEMENTARY MATERIAL}

The Supplementary Material for this article can be found online at: https://www.frontiersin.org/articles/10.3389/fmed. 2021.778536/full\#supplementary-material a prospective observational cohort study. Intensive Care Med Exp. (2019) 7:26. doi: 10.1186/s40635-019-0252-3

14. Rovas A, Seidel LM, Vink H, Pohlkotter T, Pavenstadt H, Ertmer $\mathrm{C}$, et al. Association of sublingual microcirculation parameters and endothelial glycocalyx dimensions in resuscitated sepsis. Crit Care. (2019) 23:260. doi: 10.1186/s13054-019-2542-2

15. Weiss SL, Peters MJ, Alhazzani W, Agus MSD, Flori HR, Inwald DP, et al. Surviving sepsis campaign international guidelines for the management of septic shock and sepsis-associated organ dysfunction in children. Intensive Care Med. (2020) 46:10-67. doi: 10.1007/s00134-019-05878-6

16. Zuccari S, Damiani E, Domizi R, Scorcella C, D’Arezzo M, Carsetti $\mathrm{A}$, et al. Changes in cytokines, haemodynamics and microcirculation in patients with sepsis/septic shock undergoing continuous renal replacement therapy and blood purification with CytoSorb. Blood Purif. (2020) 49:10713. doi: $10.1159 / 000502540$

17. McHale TM, Garciarena CD, Fagan RP, Smith SGJ, Martin-Loches I, Curley GF, et al. Inhibition of vascular endothelial cell leak following Escherichia coli attachment in an experimental model of sepsis. Crit Care Med. (2018) 46:e805-10. doi: 10.1097/CCM.0000000000003219

18. Quinto BM, Iizuka IJ, Monte JC, Santos BF, Pereira V, Durao MS, et al. TNF-alpha depuration is a predictor of mortality in critically ill patients under continuous veno-venous hemodiafiltration treatment. Cytokine. (2015) 71:255-60. doi: 10.1016/j.cyto.2014.10.024

19. Tolwani AJ, Wille KM. Anticoagulation for continuous renal replacement therapy. Semin Dial. (2009) 22:141-5. doi: 10.1111/j.1525-139X.2008.00545.x

20. Johnson AE, Pollard TJ, Shen L, Lehman LW, Feng M, Ghassemi M, et al. MIMIC-III, a freely accessible critical care database. Sci Data. (2016) 3:160035. doi: 10.1038/sdata.2016.35

21. Seymour CW, Liu VX, Iwashyna TJ, Brunkhorst FM, Rea TD, Scherag A, et al. Assessment of clinical criteria for sepsis: for the third international consensus definitions for sepsis and septic shock (Sepsis-3). JAMA. (2016) 315:762-74. doi: 10.1001/jama.2016.0288

22. Singer M, Deutschman CS, Seymour CW, Shankar-Hari M, Annane D, Bauer $\mathrm{M}$, et al. The third international consensus definitions for sepsis and septic shock (Sepsis-3). JAMA. (2016) 315:801-10. doi: 10.1001/jama.2016.0287

23. Johnson AEW, Aboab J, Raffa JD, Pollard TJ, Deliberato RO, Celi LA, et al. A comparative analysis of sepsis identification methods in an electronic database. Crit Care Med. (2018) 46:494-9. doi: 10.1097/CCM.0000000000002965

24. Rhodes A, Evans LE, Alhazzani W, Levy MM, Antonelli M, Ferrer R, et al. Surviving sepsis campaign: international guidelines for management of sepsis and septic shock: 2016. Intensive Care Med. (2017) 43:304-77. doi: 10.1007/s00134-017-4683-6

25. Heung M, Bagshaw SM, House AA, Juncos LA, Piazza R, Goldstein SL. CRRTnet: a prospective, multi-national, observational study of continuous renal replacement therapy practices. BMC Nephrol. (2017) 18:222. doi: 10.1186/s12882-017-0650-2 
26. Xie J, Wang H, Kang Y, Zhou L, Liu Z, Qin B, et al. The epidemiology of sepsis in Chinese ICUs: a national cross-sectional survey. Crit Care Med. (2020) 48:e209-18. doi: 10.1097/CCM.0000000000004155

27. Tang X, Shao L, Dou J, Zhou Y, Chen M, Cui Y, et al. Fibrinogen as a prognostic predictor in pediatric patients with sepsis: a database study. Med Inflamm. (2020) 2020:9153620. doi: 10.1155/2020/9153620

28. Lin L, Wang X, Ren J, Sun Y, Yu R, Li K, et al. Risk factors and prognosis for COVID-19-induced acute kidney injury: a meta-analysis. BMJ Open. (2020) 10:e042573. doi: 10.1136/bmjopen-2020-042573

29. Miao H, Shi J, Wang C, Lu G, Zhu X, Wang Y, et al. Continuous renal replacement therapy in pediatric severe sepsis: a propensity score-matched prospective multicenter cohort study in the PICU. Crit Care Med. (2019) 47:e806-13. doi: 10.1097/CCM.0000000000003901

30. Perez-Fernandez X, Sabater-Riera J, Sileanu FE, Vazquez-Reveron J, BallusNoguera J, Cardenas-Campos P, et al. Clinical variables associated with poor outcome from sepsis-associated acute kidney injury and the relationship with timing of initiation of renal replacement therapy. J Crit Care. (2017) 40:154-60. doi: 10.1016/j.jcrc.2017.03.022

31. Park JT, Lee H, Kee YK, Park S, Oh HJ, Han SH, et al. High-dose versus conventional-dose continuous venovenous hemodiafiltration and patient and kidney survival and cytokine removal in sepsis-associated acute kidney injury: a randomized controlled trial. Am J Kidney Dis. (2016) 68:599-608. doi: 10.1053/j.ajkd.2016.02.049

32. Romagnoli S, Ricci Z, Ronco C. CRRT for sepsis-induced acute kidney injury. Curr Opin Crit Care. (2018) 24:483-92. doi: 10.1097/MCC.0000000000000544

33. Miao H, Wang F, Xiong X, Wang C, Zhang Y. Clinical benefits of high-volume hemofiltration in critically ill pediatric patients with severe sepsis: a retrospective cohort study. Blood Purif. (2018) 45:1827. doi: 10.1159/000481249

34. Santiago MJ, Lopez-Herce J, Urbano J, Solana MJ, del Castillo J, Ballestero $\mathrm{Y}$, et al. Clinical course and mortality risk factors in critically ill children requiring continuous renal replacement therapy. Intensive Care Med. (2010) 36:843-9. doi: 10.1007/s00134-010-1858-9
35. Guru PK, Singh TD, Akhoundi A, Kashani KB. Association of thrombocytopenia and mortality in critically ill patients on continuous renal replacement therapy. Nephron. (2016) 133:175-82. doi: 10.1159/000 447543

36. Yoon J, Kim Y, Yim H, Cho YS, Kym D, Hur J, et al. Analysis of prognostic factors for acute kidney injury with continuous renal replacement therapy in severely burned patients. Burns. (2017) 43:141826. doi: 10.1016/j.burns.2017.03.015

37. Zhang Q, Fei Y, Jiang L. [Risk factors for mortality in intensive care unit patients with sepsis combined with acute kidney injury after continuous renal replacement therapy: secondary analysis of the data from a multicenter observational study]. Zhonghua Wei Zhong Bing Ji Jiu Yi Xue. (2019) 31:155-9. doi: 10.3760/cma.j.issn.2095-4352.2019. 02.007

Conflict of Interest: The authors declare that the research was conducted in the absence of any commercial or financial relationships that could be construed as a potential conflict of interest.

Publisher's Note: All claims expressed in this article are solely those of the authors and do not necessarily represent those of their affiliated organizations, or those of the publisher, the editors and the reviewers. Any product that may be evaluated in this article, or claim that may be made by its manufacturer, is not guaranteed or endorsed by the publisher.

Copyright (c) 2022 Wang, Zheng, Wang, Zou and Zhang. This is an open-access article distributed under the terms of the Creative Commons Attribution License (CC $B Y)$. The use, distribution or reproduction in other forums is permitted, provided the original author(s) and the copyright owner(s) are credited and that the original publication in this journal is cited, in accordance with accepted academic practice. No use, distribution or reproduction is permitted which does not comply with these terms. 